\title{
On Monotonic and Nonnegative Solutions of a Nonlinear Volterra-Stieltjes Integral Equation
}

\author{
Tomasz Zając \\ Department of Mathematics, Rzeszów University of Technology, Aleja Powstańców Warszawy 12, 35-959 Rzeszów, Poland \\ Correspondence should be addressed to Tomasz Zając; tzajac@prz.edu.pl
}

Received 11 April 2014; Accepted 27 May 2014; Published 9 July 2014

Academic Editor: Józef Banaś

Copyright (C) 2014 Tomasz Zając. This is an open access article distributed under the Creative Commons Attribution License, which permits unrestricted use, distribution, and reproduction in any medium, provided the original work is properly cited.

\begin{abstract}
We study the existence of monotonic and nonnegative solutions of a nonlinear quadratic Volterra-Stieltjes integral equation in the space of real functions being continuous on a bounded interval. The main tools used in our considerations are the technique of measures of noncompactness in connection with the theory of functions of bounded variation and the theory of Riemann-Stieltjes integral. The obtained results can be easily applied to the class of fractional integral equations and Volterra-Chandrasekhar integral equations, among others.
\end{abstract}

\section{Introduction}

The aim of this paper is to study of monotonic and nonnegative solutions of the nonlinear quadratic Volterra-Stieltjes integral equation having the form

$$
x(t)=\left(F_{1} x\right)(t)+\left(F_{2} x\right)(t) \int_{0}^{t} u(t, \tau,(T x)(\tau)) d_{\tau} g(t, \tau),
$$

where $t \in[a, b]$ and $F_{1}, F_{2}$ are superposition operators defined on the function space $C[a, b]$. The precise definitions will be given later. We show the existence of such solutions of the previous equation under some reasonable and handy assumptions. In our considerations, we use the technique associated with measures of noncompactness and the Riemann-Stieltjes integral with a kernel depending on two variables. Moreover, the theory of functions of bounded variation is also employed.

The main result of the paper is contained in Theorem 8 . That theorem covers, as particular cases, the classical Volterra integral equation, the integral equation of fractional order, and the Volterra counterpart of the famous integral equation of Chandrasekhar type. It is worth pointing out that differential and integral equations of fractional order create an important branch of nonlinear analysis and the theory of integral equations. Moreover, these equations have found a lot of applications connected with real world problems. Integral equations of Chandrasekhar type can be often encountered in several applications as well.

This paper can be considered as a continuation of $[1,2]$ (cf. also [3-5]).

\section{Preliminaries}

At the beginning, we provide some basic facts concerning functions of bounded variation and the Riemann-Stieltjes integral. We refer to [6] or [7] for more information about this subject. Assume that $x$ is a real function defined on the interval $[a, b]$. The symbol $\bigvee_{a}^{b} x$ stands for the variation of the function $x$ on the interval $[a, b]$. In case of a function $u(t, \tau)=u: A \rightarrow \mathbb{R}$, where $A \subset \mathbb{R}^{2}$, the symbol $\bigvee_{\tau=p}^{q} u(t, \tau)$ denotes the variation of the function $\tau \rightarrow u(t, \tau)$ on the interval $[p, q]$ which is contained in the domain of this function, where the variable $t$ is fixed. Further, assume that $x, \varphi$ are given real functions defined on the interval $[a, b]$. Then, under some additional conditions imposed on $x$ and $\varphi$, we can define the Riemann-Stieltjes integral

$$
\int_{a}^{b} x(t) d \varphi(t)
$$


of the function $x$ with respect to the function $\varphi$. In such a case, we say that $x$ is integrable in the Riemann-Stieltjes sense on the interval $[a, b]$ with respect to $\varphi$.

Now, we recall two useful properties of the RiemannStieltjes integral, which will be employed in the sequel.

Theorem 1. (a) If $x$ is a continuous function and $\varphi$ is a function of bounded variation on the interval $[a, b]$, then $x$ is RiemannStieltjes integrable on $[a, b]$ with respect to $\varphi$.

(b) Suppose that $x_{1}$ and $x_{2}$ are functions being RiemannStieltjes integrable on the interval $[a, b]$ with respect to $a$ nondecreasing function $\varphi$ and $x_{1}(t) \leq x_{2}(t)$, for $t \in[a, b]$. Then,

$$
\int_{a}^{b} x_{1}(t) d \varphi(t) \leq \int_{a}^{b} x_{2}(t) d \varphi(t) .
$$

In what follows we will use the Riemann-Stieltjes integral of the form

$$
\int_{a}^{b} x(\tau) d_{\tau} g(t, \tau),
$$

where the symbol $d_{\tau}$ indicates the integration with respect to the variable $\tau$ and $t$ is fixed. Let us mention that, in some situations, lower and upper limit of the integration can also depend upon the variable $t$.

Now, we deal with the discussion of basic facts connected with measures of noncompactness. We refer to [8] (see also [9]) for a more detailed discussion. Assume that $E$ is a real Banach space. Denote by $B(x, r)$ the closed ball centered at $x$ and with radius $r$. Instead of $B(0, r)$, we will write $B_{r}$. If $X$ is a subset of $E$, then the symbols $\bar{X}$ and Conv $X$ denote the closure and convex closed hull of the set $X$, respectively. Further, denote by $\mathfrak{M}_{E}$ the family of all nonempty and bounded subsets of $E$. The symbol $\mathfrak{N}_{E}$ stands for the subfamily of $\mathfrak{M}_{E}$ consisting of all relatively compact sets. We will accept the following definition of a measure of noncompactness.

Definition 2. A mapping $\mu: \mathfrak{M}_{E} \rightarrow \mathbb{R}_{+}=[0,+\infty)$ will be called a measure of noncompactness in the space $E$ if it satisfies the following conditions:

(1) the family $\operatorname{ker} \mu=\left\{X \in \mathfrak{M}_{E}: \mu(X)=0\right\}$ is nonempty and $\operatorname{ker} \mu \subset \mathfrak{N}_{E}$;

(2) $X \subset Y \Rightarrow \mu(X) \leq \mu(Y)$;

(3) $\mu(X)=\mu(\bar{X})=\mu(\operatorname{Conv} X)$;

(4) $\mu(\lambda X+(1-\lambda) Y) \leq \lambda \mu(X)+(1-\lambda) \mu(Y)$, for $\lambda \in[0,1]$;

(5) if $\left(X_{n}\right)$ is a sequence of closed sets belonging to $\mathfrak{M}_{E}$ such that $X_{n+1} \subset X_{n}$, for $n=1,2, \ldots$, and if $\lim _{n \rightarrow \infty}$ $\mu\left(X_{n}\right)=0$, then the intersection $X_{\infty}=\bigcap_{n=1}^{\infty} X_{n}$ is nonempty.

An important example of a measure of noncompactness is the Hausdorff measure of noncompactness defined by the formula

$$
\begin{array}{r}
\chi(X)=\inf \{\varepsilon>0: X \text { has a finite } \varepsilon-\text { net in } E\}, \\
\qquad X \in \mathfrak{M}_{E} .
\end{array}
$$

The key role in our studies will be played by Darbo's fixed point theorem.

Theorem 3. Let $\Omega$ be a nonempty, bounded, closed, and convex subset of the space $E$ and let $Q: \Omega \rightarrow \Omega$ be a continuous transformation. Assume that there exists a constant $k \in[0,1)$ such that $\mu(Q X) \leq k \mu(X)$ for any nonempty subset $X$ of $\Omega$. Then, $Q$ has at least one fixed point in the set $\Omega$. Moreover, the set Fix $Q$ of all fixed points of $Q$ belonging to $\Omega$ is a member of the family $\operatorname{ker} \mu$.

The considerations in this paper will be placed in the Banach space $C[a, b]$ consisting of all real functions defined and continuous on the bounded interval $[a, b]$ with the standard maximum norm.

Finally, we turn our attention to the superposition (or Nemytskii) operator which appears very frequently in nonlinear analysis. We refer to monographs $[6,10]$ for detailed information covering the properties of this operator. To define the operator in question, suppose that $f:[a, b] \times \mathbb{R} \rightarrow$ $\mathbb{R}$ is a given function. For any function $x(t)=x:[a, b] \rightarrow \mathbb{R}$, we can define the function $F x$ by putting

$$
(F x)(t)=f(t, x(t)), \quad t \in[a, b] .
$$

The operator $F$ defined in such a way is called the superposition operator generated by the function $f$.

\section{Main Result}

In this section, we will investigate the nonlinear quadratic Volterra-Stieltjes integral equation which has the form

$$
\begin{aligned}
x(t)= & f_{1}(t, x(t))+f_{2}(t, x(t)) \\
& \times \int_{0}^{t} u(t, \tau,(T x)(\tau)) d_{\tau} g(t, \tau), \quad t \in I=[0, M],
\end{aligned}
$$

where $M>0$ is fixed number. Obviously, in our further considerations the interval $I=[0, M]$ can be replaced by any interval $[a, b]$. We look for monotonic and nonnegative solutions of this equation in the space $C[0, M]$. In our study, we will need some results obtained in $[1,2]$.

At the beginning, let us consider the following conditions.

(i) The functions $f_{i}: I \times \mathbb{R} \rightarrow \mathbb{R}(i=1,2)$ are continuous and there exist nondecreasing functions $k_{i}: \mathbb{R}_{+} \rightarrow \mathbb{R}_{+}$such that

$$
\left|f_{i}(t, x)-f_{i}(t, y)\right| \leq k_{i}(r)|x-y| \quad(i=1,2),
$$

for any $t \in I$ and for all $x, y \in[-r, r]$, where $r \geq 0$ is an arbitrary fixed number.

Observe that, on the basis of the above condition, we may define the finite constants $\overline{F_{1}}, \overline{F_{2}}$ by putting

$$
\overline{F_{i}}=\max \left\{\left|f_{i}(t, 0)\right|: t \in I\right\} \quad(i=1,2) .
$$

Let $\Delta_{M}$ denote the following triangle:

$$
\Delta_{M}=\left\{(t, \tau) \in \mathbb{R}^{2}: 0 \leq \tau \leq t \leq M\right\} .
$$


(ii) The function $u: \Delta_{M} \times \mathbb{R} \rightarrow \mathbb{R}$ is continuous. Moreover, there exists a continuous function $\Phi: \mathbb{R}_{+} \rightarrow$ $\mathbb{R}_{+}$such that

$$
|u(t, \tau, x)| \leq \Phi(|x|),
$$

for all $(t, \tau) \in \Delta_{M}$ and $x \in \mathbb{R}$.

(iii) The function $g: \Delta_{M} \rightarrow \mathbb{R}$ is continuous with respect to the variable $\tau$ on the interval $[0, t]$, where $t \in I$ is fixed.

(iv) For any $t \in I$, the function $\tau \rightarrow g(t, \tau)$ is of bounded variation on the interval $[0, t]$.

(v) For each $\varepsilon>0$, there exists $\delta>0$ such that, for all $t, s \in I$ and $|s-t| \leq \delta$, the following inequality holds

$$
\bigvee_{\tau=0}^{\min \{t, s\}}[g(s, \tau)-g(t, \tau)] \leq \varepsilon
$$

Remark 4. It can be shown (see $[1,2])$ that the constant

$$
K=\max \left\{\bigvee_{\tau=0}^{t} g(t, \tau): t \in I\right\}
$$

is well defined and finite.

(vi) The operator $T: C(I) \rightarrow C(I)$ is continuous and there exists a nondecreasing function $\Psi: \mathbb{R}_{+} \rightarrow \mathbb{R}_{+}$ such that $\|T x\| \leq \Psi(\|x\|)$, for any $x \in C(I)$.

(vii) There exists a positive real number $r_{0}$ which satisfies the inequalities

$$
\begin{gathered}
r k_{1}(r)+\overline{F_{1}}+K\left(r k_{2}(r)+\overline{F_{2}}\right) \Phi(\Psi(r)) \leq r, \\
k_{1}(r)+K k_{2}(r) \Phi(\Psi(r))<1 .
\end{gathered}
$$

Remark 5. Observe that if $r_{0}$ is a positive solution of the first inequality from condition (vii) and if one of the terms $\overline{F_{1}}$ and $K \overline{F_{2}} \Phi\left(\Psi\left(r_{0}\right)\right)$ does not vanish, then the second inequality from (vii) is automatically satisfied.

Now, let us consider the operators $F_{i}(i=1,2), U$, and $V$ defined on the space $C(I)$ by the following formulas:

$$
\begin{gathered}
\left(F_{i} x\right)(t)=f_{i}(t, x(t)) \quad(i=1,2), \\
(U x)(t)=\int_{0}^{t} u(t, \tau,(T x)(\tau)) d_{\tau} g(t, \tau), \\
(V x)(t)=\left(F_{1} x\right)(t)+\left(F_{2} x\right)(t)(U x)(t) .
\end{gathered}
$$

Theorem 6. Let conditions (i)-(vii) hold. Then, the operator $V_{\mid B_{r_{0}}}: B_{r_{0}} \rightarrow B_{r_{0}}$ is well defined and continuous and has at least one fixed point, which gives that (7) has at least one solution in the ball $B_{r_{0}}$, where $r_{0}$ is a number appearing in condition (vii).

The basic idea of the proof of Theorem 6 is to study behaviour of the operator $V$ with respect to the Hausdorff measure of noncompactness in connection with Theorem 3.
Remark 7. Additionally, all solutions of (7) from the ball $B_{r_{0}}$ are equicontinuous. This observation results directly from the Arzela-Ascoli theorem and Theorem 3.

We can now formulate our main result about monotonicity and nonnegativity of the solutions of (7). In our study, we will consider the following conditions.

$\left(i^{\prime}\right)$ The functions $f_{i}: I \times \mathbb{R} \rightarrow \mathbb{R}(i=1,2)$ are such that

(1) $f_{i}\left(I \times \mathbb{R}_{+}\right) \subset \mathbb{R}_{+}$;

(2) the function $t \rightarrow f_{i}(t, x)$ is nondecreasing on $I$, for any fixed $x \in \mathbb{R}_{+}$

(3) the function $x \rightarrow f_{i}(t, x)$ is nondecreasing on $\mathbb{R}_{+}$, for any fixed $t \in I$.

(ii') (a) The function $u: \Delta_{M} \times \mathbb{R} \rightarrow \mathbb{R}$ is such that

(1) $u\left(\Delta_{M} \times \mathbb{R}_{+}\right) \subset \mathbb{R}_{+}$;

(2) the function $t \rightarrow u(t, \tau, x)$ is nondecreasing on $[\tau, M]$, for any fixed $\tau \in I$ and $x \in \mathbb{R}_{+}$;

(3) for each $t, s \in I$ such that $t<s$, the function $\tau \rightarrow g(s, \tau)-g(t, \tau)$ is nondecreasing on $[0, t]$;

(4) for any function $x \in B_{r_{0}}$ which is nonnegative and nondecreasing on $I$, the function $T x$ is nonnegative on $I$, where $r_{0}$ is a number appearing in condition (vii)

Or

(b) The function $u: \Delta_{M} \times \mathbb{R} \rightarrow \mathbb{R}$ is such that

(1) $u\left(\Delta_{M} \times \mathbb{R}_{+}\right) \subset \mathbb{R}_{+}$;

(2) the function $t \rightarrow u(t, \tau, x)$ is nondecreasing on $[\tau, M]$, for any fixed $\tau \in I$ and $x \in \mathbb{R}_{+}$;

(3) the function $\tau \rightarrow u(t, \tau, x)$ is nondecreasing on $[0, t]$, for any fixed $t \in I$ and $x \in \mathbb{R}_{+}$;

(4) the function $x \rightarrow u(t, \tau, x)$ is nondecreasing on $\mathbb{R}_{+}$for any fixed $(t, \tau) \in \Delta_{M} ;$

(5) for each $t, s \in I$ such that $t<s$, the function $\tau \rightarrow g(s, \tau)-g(t, \tau)$ is nondecreasing on $[0, t]$;

(6) $g(s, s)-g(t, t)+g(t, 0)-g(s, 0) \geq 0$;

(7) for any function $x \in B_{r_{0}}$ which is nonnegative and nondecreasing on $I$, the function $T x$ is nonnegative and nondecreasing on $I$, where $r_{0}$ is a number appearing in condition (vii).

(iii $\left.^{\prime}\right)$ For each $t \in I$ the function $\tau \rightarrow g(t, \tau)$ is nondecreasing on $[0, t]$.

The following theorem is a completion of Theorem 6 .

Theorem 8. Suppose that conditions (i)-(vii) and $\left(i^{\prime}\right)-\left(i i i^{\prime}\right)$ are fulfilled. Then, (7) has at least one solution in $B_{r_{0}}$ which is nonnegative and nondecreasing, where $r_{0}$ is a number appearing in condition (vii). 
Proof. Let $B_{r_{0}}^{+}$denote set of all nonnegative and nondecreasing functions from the ball $B_{r_{0}}$. It is clear that $B_{r_{0}}^{+}$is nonempty, bounded, closed, and convex. From Theorem 6, we conclude that the operator $V_{\mid B_{r_{0}}^{+}}$is continuous. We show that $V\left(B_{r_{0}}^{+}\right) \subset$ $B_{r_{0}}^{+}$. To this end, fix $x \in B_{r_{0}}^{+}$and take $t, s \in I$ such that $s>t$. Since $\left(F_{i} x\right)(t)=f_{i}(t, x(t)) \geq 0$ and

$$
\begin{aligned}
\left(F_{i} x\right)(s)-\left(F_{i} x\right)(t) & =f_{i}(s, x(s))-f_{i}(t, x(t)) \\
& \geq f_{i}(t, x(s))-f_{i}(t, x(t)) \geq 0,
\end{aligned}
$$

we obtain $F_{i}\left(B_{r_{0}}^{+}\right) \subset B_{r_{0}}^{+}$, for $i=1,2$.

It is easily seen that $(U x)(t) \geq 0$ so it suffices to check monotonicity of the operator $U$. We get

$$
\begin{aligned}
(U x) & (s)-(U x)(t) \\
= & \int_{0}^{s} u(s, \tau,(T x)(\tau)) d_{\tau} g(s, \tau) \\
& -\int_{0}^{t} u(t, \tau,(T x)(\tau)) d_{\tau} g(t, \tau) \\
= & \int_{0}^{t} u(s, \tau,(T x)(\tau)) d_{\tau} g(s, \tau) \\
& +\int_{t}^{s} u(s, \tau,(T x)(\tau)) d_{\tau} g(s, \tau) \\
& -\int_{0}^{t} u(t, \tau,(T x)(\tau)) d_{\tau} g(t, \tau) \\
\geq & \int_{0}^{t} u(s, \tau,(T x)(\tau)) d_{\tau} g(s, \tau) \\
& +\int_{t}^{s} u(s, \tau,(T x)(\tau)) d_{\tau} g(s, \tau) \\
& -\int_{0}^{t} u(s, \tau,(T x)(\tau)) d_{\tau} g(t, \tau) \\
& +\int_{t}^{s} u(s, \tau,(T x)(\tau)) d_{\tau} g(s, \tau) \\
& \int_{0}^{t} u(s, \tau,(T x)(\tau)) d_{\tau}[g(s, \tau)-g(t, \tau)]
\end{aligned}
$$

Further proving process depends on which of conditions (ii $\left.{ }^{\prime}(\mathrm{a})\right)$ or $\left(\mathrm{ii}^{\prime}(\mathrm{b})\right)$ is satisfied.

Assume that condition (ii' (a)) holds. Then based on Theorem 1, the two last integrals in estimation (17) are nonnegative and indeed $(U x)(s)-(U x)(t) \geq 0$.

Now, assume that condition (ii $\left.{ }^{\prime}(\mathrm{b})\right)$ is satisfied. Coming back to estimation (17), we obtain

$$
\begin{aligned}
& (U x)(s)-(U x)(t) \\
& \geq \int_{0}^{t} u(s, t,(T x)(t)) d_{\tau}[g(s, \tau)-g(t, \tau)] \\
& \quad+\int_{t}^{s} u(s, t,(T x)(t)) d_{\tau} g(s, \tau)
\end{aligned}
$$

$$
\begin{gathered}
=u(s, t,(T x)(t))[g(s, t)-g(t, t)-(g(s, 0)-g(t, 0)) \\
+g(s, s)-g(s, t)] \\
=u(s, t,(T x)(t))[g(s, s)-g(t, t)+g(t, 0) \\
-g(s, 0)] \geq 0
\end{gathered}
$$

and, consequently, $U\left(B_{r_{0}}^{+}\right) \subset B_{r_{0}}^{+}$. Finally, we have $V\left(B_{r_{0}}^{+}\right) \subset$ $B_{r_{0}}^{+}$. Using Theorems 3 and 6 , we obtain the existence of a fixed point of the operator $V$ in $B_{r_{0}}^{+}$. This means that (7) has at least one nonnegative and nondecreasing solution in $B_{r_{0}}$, and the proof is complete.

Remark 9. It can be shown (see for instance [1]) that if the function $g: \Delta_{M} \rightarrow \mathbb{R}$ is continuous on the triangle $\Delta_{M}$ and for arbitrarily fixed $t, s \in I$ such that $t<s$, the function $\tau \rightarrow g(s, \tau)-g(t, \tau)$ is monotonic (nondecreasing or nonincreasing) on the interval $[0, t]$; then $g$ satisfies condition (v).

\section{Applications and an Example}

The topic of this section is to present some applications of Theorem 8 in the situation of the classical integral equations.

Let us consider the equation

$$
\begin{aligned}
x(t)= & f_{1}(t, x(t))+\frac{\tilde{f}_{2}(t, x(t))}{\Gamma(\alpha)} \\
& \times \int_{0}^{t} \frac{u(t, \tau,(T x)(\tau))}{(t-\tau)^{1-\alpha}} d \tau, \quad t \in I,
\end{aligned}
$$

where $\Gamma$ denotes the Euler gamma function and $\alpha>0$. It is the well-known integral equation of fractional order. If we take on the set $\Delta_{M}$ the function $g$ defined by

$$
g(t, \tau)=\frac{1}{\alpha}\left[t^{\alpha}-(t-\tau)^{\alpha}\right],
$$

then it is easy to check that (19) is a special case of (7). Using Remark 9 and the standard methods of differential calculus, we can show that the function $g$ satisfies conditions (iii) $-(v)$, (ii'), and (iii'). Additionally, we have $K=(1 / \alpha) M^{\alpha}$, where $K$ is the constant appearing in Remark 4. Making use of the fact that $\Gamma(\alpha+1)=\alpha \Gamma(\alpha)$ for $\alpha>0$, condition (vii) in this situation takes the following form:

$\left(\right.$ vii $\left.^{*}\right)$ there exists a positive real number $r_{0}$ which satisfies the inequalities

$$
\begin{gathered}
r k_{1}(r)+\overline{F_{1}}+\frac{M^{\alpha}}{\Gamma(\alpha+1)}\left(r \widetilde{k}_{2}(r)+\widetilde{F}_{2}\right) \Phi(\Psi(r)) \leq r, \\
k_{1}(r)+\frac{M^{\alpha}}{\Gamma(\alpha+1)} \widetilde{k}_{2}(r) \Phi(\Psi(r))<1,
\end{gathered}
$$

where $\widetilde{F}_{2}=\max \left\{\left|\widetilde{f}_{2}(t, 0)\right|: t \in I\right\}$ and $\widetilde{k}_{2}$ is a function chosen for $\widetilde{f}_{2}$ based on condition (i). 
Obviously, when $\alpha=1$, (19) reduces to the classical nonlinear quadratic Volterra integral equation.

Now, let us consider the equation

$$
\begin{aligned}
x(t)= & f_{1}(t, x(t))+f_{2}(t, x(t)) \\
& \times \int_{0}^{t} \frac{t}{t+\tau} u(t, \tau,(T x)(\tau)) d \tau, \quad t \in I .
\end{aligned}
$$

It is the Volterra counterpart of the quadratic integral equation of Chandrasekhar type. This equation is also a special case of (7), in which

$$
g(t, \tau)= \begin{cases}t \ln \left(1+\frac{\tau}{t}\right), & (t, \tau) \in \Delta_{M} \backslash\{(0,0)\} \\ 0, & t=\tau=0 .\end{cases}
$$

Using, as before, Remark 9 and the standard methods of differential calculus, we can show that this function satisfies conditions (iii)-(v), ( $\left.\mathrm{ii}^{\prime}(\mathrm{a})\right)$, and ( $\left.\mathrm{iii}^{\prime}\right)$. Additionally, we have $K=M \ln 2$, where $K$ is the constant appearing in Remark 4 .

Let us observe that if we put $f_{2}(t, x) \equiv 0$ in (7), we obtain the classical functional equation of the first order on the interval $I$.

We finish by providing an example illustrating Theorem 8 .

Example 1. Let us consider the following integral equation:

$$
\begin{aligned}
x(t)= & t e^{-t}+\frac{t^{2}+x(t)}{\Gamma(2 / 3)} \\
& \times \int_{0}^{t} \frac{1}{2 \pi} \frac{\sqrt{|x(\tau)|} \operatorname{arctg}\left(4+t^{2}+\tau^{2}\right)}{\sqrt[3]{t-\tau}} d \tau, \quad t \in[0,1] .
\end{aligned}
$$

Obviously, this equation is a special case of (19) if we put $\alpha=$ $2 / 3$ and

$$
\begin{gathered}
f_{1}(t, x)=t e^{-t}, \\
\tilde{f}_{2}(f, x)=t^{2}+x, \\
u(t, \tau, x)=\frac{1}{2 \pi} \sqrt{|x|} \operatorname{arctg}\left(4+t^{2}+\tau^{2}\right), \\
T x=x .
\end{gathered}
$$

In is easy to check that conditions (i)-(vi), (i'), (ii' (b)), and (iii $^{\prime}$ ) of Theorem 8 are satisfied and $k_{1}(r)=0, \overline{F_{1}}=1 / e$, $\widetilde{k}_{2}(r)=1, \widetilde{F}_{2}=1, \Phi(r)=(1 / 4) \sqrt{r}$, and $\Psi(r)=r$. Using standard estimation $\Gamma(\alpha)>0.8856$ for $\alpha>0$ and taking $r_{0}=$ 1 , we verify that condition (vii ${ }^{*}$ ) is also satisfied. Therefore, in case of (24), we can apply Theorem 8 . This means that (24) has at least one nonnegative and nondecreasing solution belonging to the ball $B_{1}$ of the space $C[0,1]$.

\section{Conflict of Interests}

The author declares that there is no conflict of interests regarding the publication of this paper.

\section{References}

[1] J. Banaś and T. Zając, "A new approach to the theory of functional integral equations of fractional order," Journal of Mathematical Analysis and Applications, vol. 375, no. 2, pp. 375387, 2011

[2] T. Zając, "Solvability of fractional integral equations on an unbounded interval through the theory of Volterra-Stieltjes integral equations," Journal of Analysis and its Applications, vol. 33, no. 1, pp. 65-85, 2014.

[3] J. Banaś and B. Rzepka, "Monotonic solutions of a quadratic integral equation of fractional order," Journal of Mathematical Analysis and Applications, vol. 332, no. 2, pp. 1371-1379, 2007.

[4] J. Caballero, B. Lopez, J. Rocha, and K. Sadarangani, "On the existence of nondecreasing solutions of an integral equation," Journal of Mathematics and Applications, vol. 28, pp. 13-24, 2006.

[5] J. Banaś and T. Zajaç, "Solvability of a functional integral equation of fractional order in the class of functions having limits at infinity," Nonlinear Analysis: Theory, Methods \& Applications, vol. 71, no. 11, pp. 5491-5500, 2009.

[6] J. Appell, J. Banas, and N. Merentes, Bounded Variation and Around, vol. 17 of De Gruyter Studies in Nonlinear Analysis and Applications, De Gruyter, Berlin, Germany, 2013.

[7] S. Lojasiewicz, An Introduction to the Theory of Real Functions, John Wiley \& Sons, Chichester, UK, 1988.

[8] J. Banaś and K. Goebel, Measures of Noncompactness, vol. 60 of Lecture Notes in Pure and Applied Mathematics, Marcel Dekker, New York, NY, USA, 1980.

[9] J. M. Ayerbe Toledano, T. Dominguez Benavides, and G. Lopez Acedo, "Measures of non-compactness in metric fixed point theory," in Operator Theory Advances and Applications, vol. 99, Birkhauser, Basel, Switzerland, 1997.

[10] J. Appell and P. P. Zabrejko, Nonlinear Superposition Operators, vol. 95 of Cambridge Tracts in Mathematics, Cambridge University Press, Cambridge, UK, 1990. 


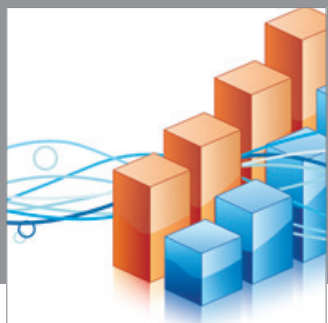

Advances in

Operations Research

mansans

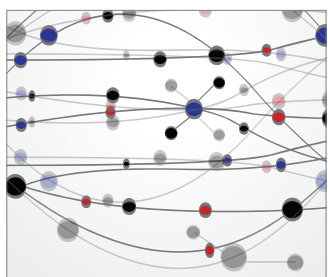

The Scientific World Journal
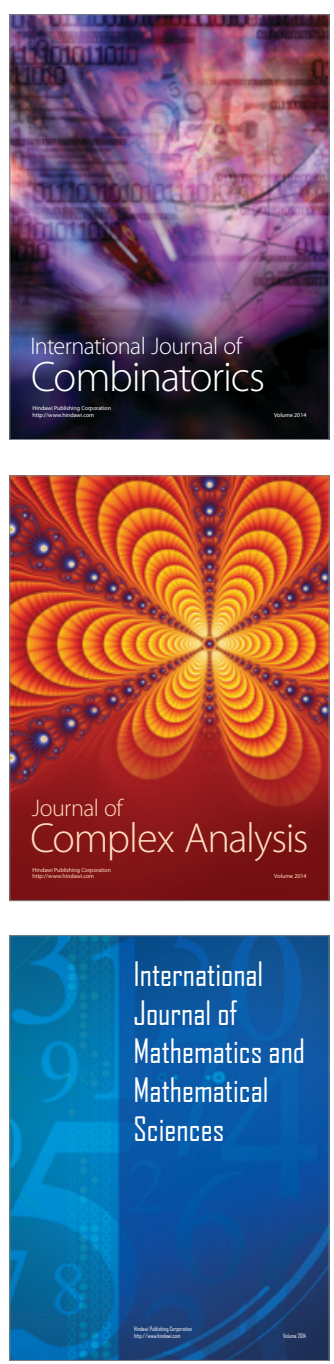
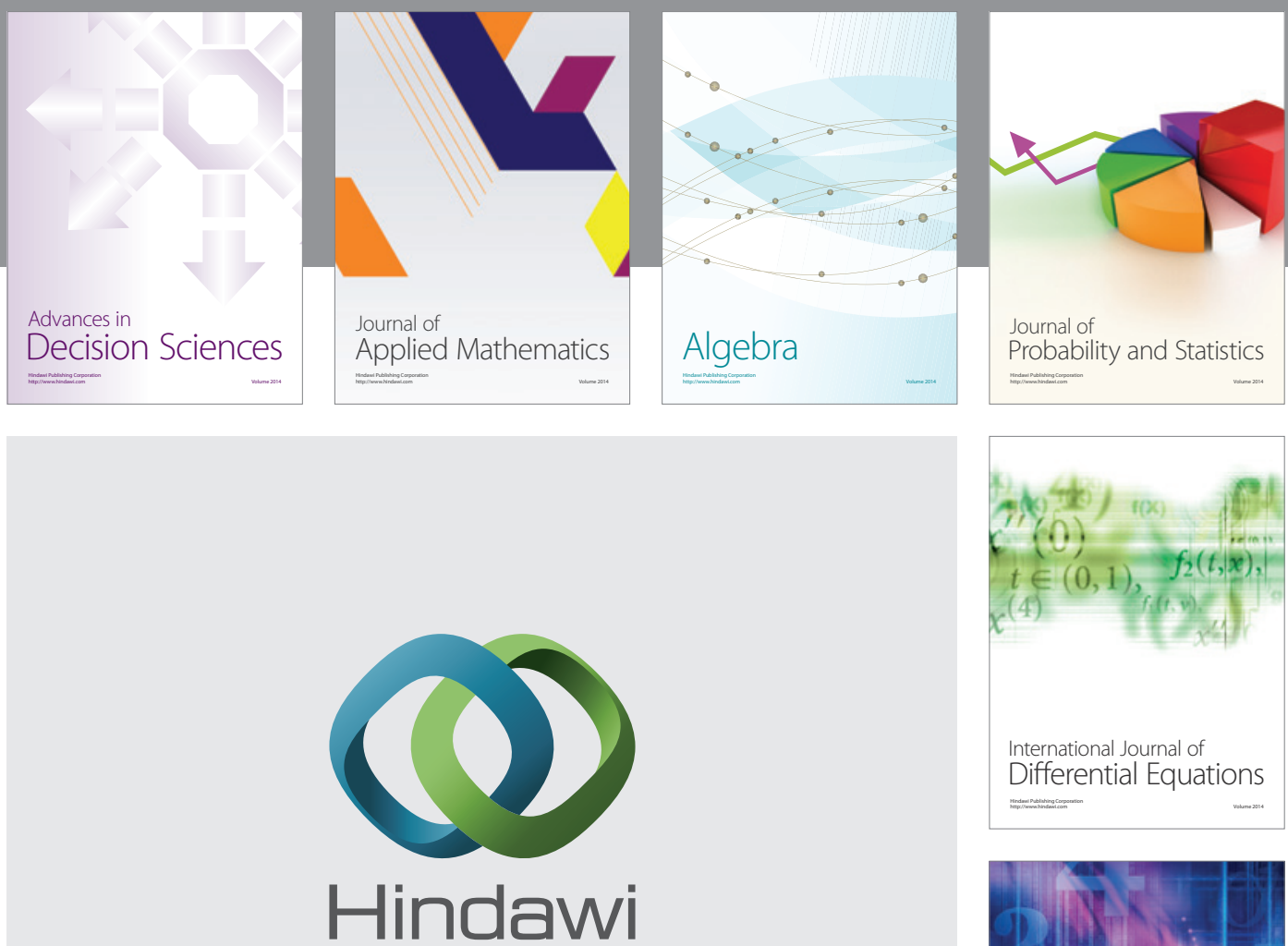

Submit your manuscripts at http://www.hindawi.com
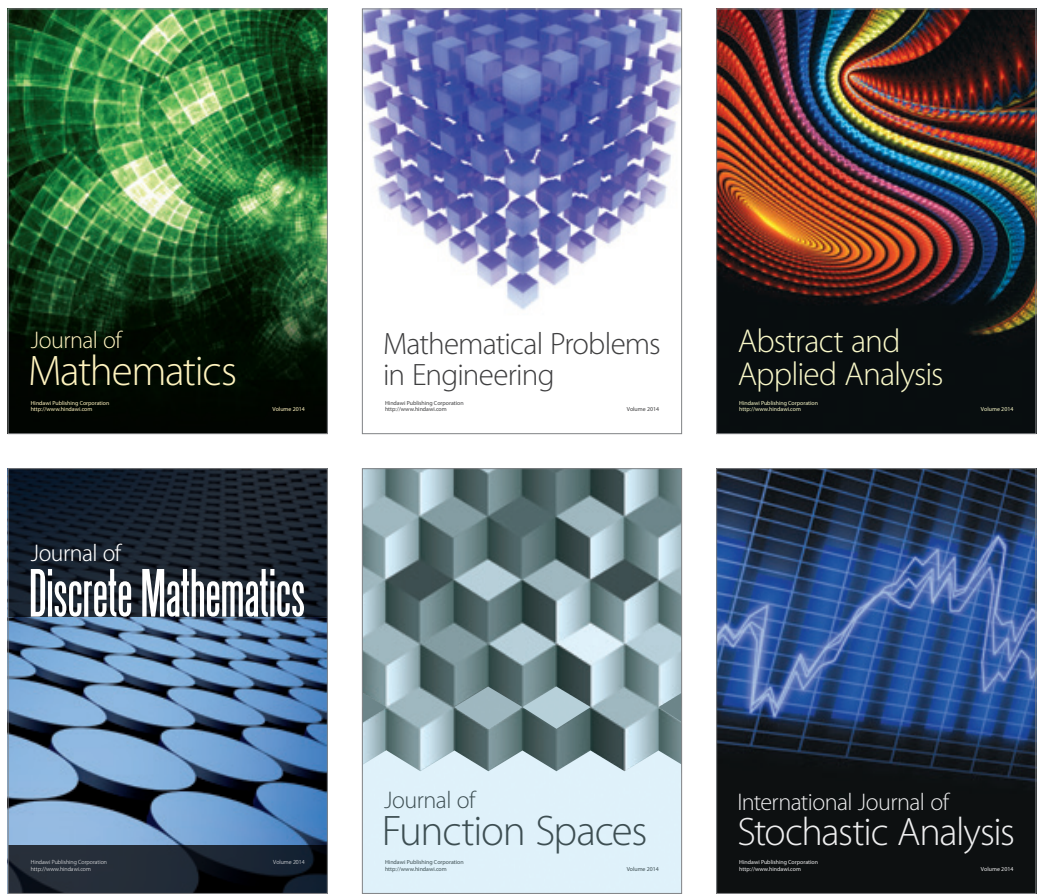

Journal of

Function Spaces

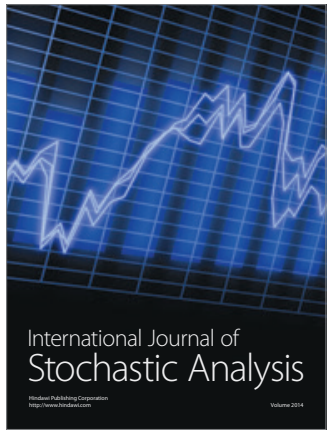

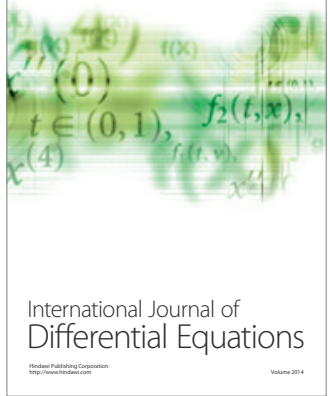
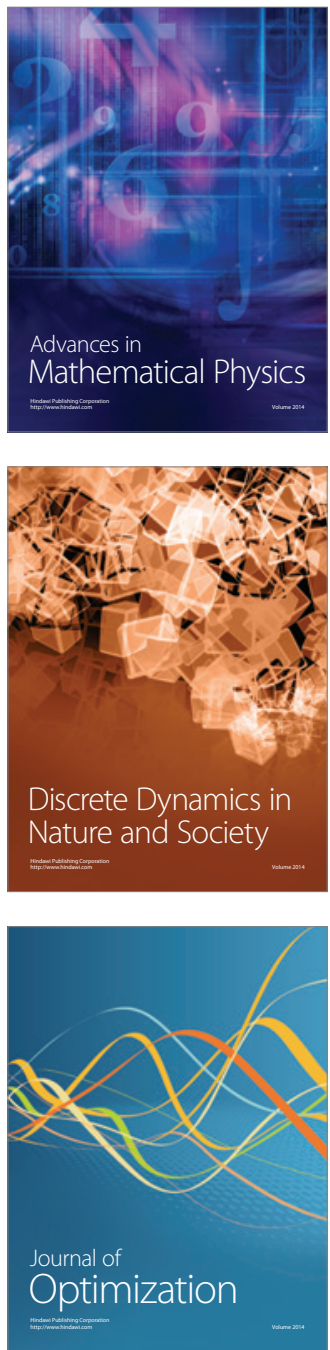